\title{
Model Based Enterprise Modeling for testing PLM Interoperability in Dynamic Manufacturing Network
}

\author{
Nicolas Figay $^{1}$, Parisa Ghodous ${ }^{2}$, Bezhad Shariat ${ }^{2}$, Ernesto Exposito ${ }^{3}$, David \\ Tchoffa $^{4}$, Lyes Kermad ${ }^{4}$, El Mouloudi Dafaoui ${ }^{4,}$ Thomas Vosgien ${ }^{5}$ \\ ${ }^{1}$ Airbus Group Innovations, Suresnes, France \\ nicolas.figay@airbus.com \\ ${ }^{2}$ University Lyon1, Lyon France \\ \{parisa.ghodous, bezhad.shariat\}@univ-lyon1fr \\ ${ }^{3}$ LAAS, Toulouse, France \\ ernesto.exposito@laas.fr \\ ${ }^{4}$ University Paris 8, Paris France \\ \{d.tchoffa, 1.kermad, e.dafaou\}@iut.univ-paris8.fr \\ ${ }^{5}$ IRT, Saclay France \\ Thomas.vosgien@irt-systemx.Fr
}

\begin{abstract}
When willing to prepare and to build operational Product Life cycle Management interoperability within a Dynamic Manufacturing Network $(\mathrm{DMN})$ in a mature digital business ecosystem such as Aeronautic, Space and Defense, the approaches proposed by the Enterprise Application Interoperability are insufficient when willing to address the existing interoperability brakes Some of these brakes have been addressed in project such as IMAGINE and SIP@SystemX, allowing to experiment innovative way of using standards based enterprise modeling and also to identify some additional gaps for applying model base enterprise modeling to PLM interoperability within a DMN. After defining the business and the scientific contexts, the paper describes this new approach which consists in federating the usage of several PLM, Business, Information and ICT standard through the usage of an enterprise modeling standardized language, ArchiMate, and associated modeling tool Archi created using ArchiMate as an EMF DSL. The defined methodology is based on producing a set of DMN blueprints and associated templates. Then, through model to model transformation, other more detailed models using more specialized languages are created and used for software component generation and deployment enterprise hub platform based on standards. Using the methodology, the associated framework and the developed resulting from our research activity, we are now able to prepare and build interoperability within a DMN. Ability of preserving investment performed with the legacy and reducing risks associated to future evolution was demonstrated through IMAGINE Aeronautic Lab experimentation within SIP. Such experimentation also highlighted some issues related to model based engineering in such a context, and allowed identifying needs for new extensions of the federative PLM interoperability framework for Collaborative Networked Product Development initiated during the ATHENA project. It will be addressed in future work.
\end{abstract}


Keywords: Model based enterprise modeling, PLM, Interoperability, standards, cloud, simulation, MDA.

\section{Introduction}

The approach described in this paper results from several successive research projects, each of them followed by operational projects allowing to assess the results and to provide new identified gaps and challenges for the next research projects. The approach, aiming at building continuous interoperability, has been developed supporting successively different industrial drivers in the PLM area: concurrent engineering, sharing of digital mock-up units distributed between heterogeneous tools, networked collaborative product development, enterprise technical applications interoperability, DMN and factory of the future. It is consequently important reminding in the introduction business and research context in order clearly understanding the challenges addressed.

\subsection{Business contexts}

Nowadays in order to remain competitive and within a global economic context where the complexity of the products is still increasing, enterprises have been developing new approaches the last years, in particular the Product Lifecycle Management (PLM) approach. [1] defines PLM as a strategic approach aiming to put in place appropriate processes related to production and consumption of data describing the product, through the different phases of the lifecycle of manufactured products and within the supply chain.

Along the lifecycle of the product and during the different PLM phases, the processes (e.g. Product Design Process) of the different enterprise functions (e.g. Design Function attributed to the Design Office) are supported by different PLM solutions constituting the Information System (IS): Product Data Management (PDM) system for the design office, Enterprise Resource Planning (ERP) systems and Manufacturing Execution System (MES) for production, Customer Service and Support (CSS) systems for support, etc. Such systems encompass processes, methods and tools which are today systematically computer aided, using software products deployed on Information and Communication technological infrastructure. A PLM application is realized by one or several instances of software products deployed in operational and technical environments PLM. PLM solutions include PLM Hubs (e.g. Boost Aerospace) which aim at interconnecting PLM applications and processes of partners collaborating around products. The need for governed standards in digital business ecosystem was identified by mature communities (e.g. eHealth in Australia working around NETHA or Automotive around VDA) for preparing and building operational interoperability. But PLM standardization governance organizations, such as ASD SSG for European Aeronautic, Space and Defense domain, are facing difficulties when elected eBusiness PLM standards and associated PLM standardization enterprise policies have to be applied in the enterprises. Different brakes exist for implementing and applying standards, which are politic, organizational or technical. 
Scientific gaps also exist for being able to properly establish continuous PLM interoperability, due to the complexity of complex systems of systems which are to be considered for supporting DMN in a continuously evolving environment.

\subsection{Research context}

To deal with the complexity of PLM and required continuous interoperability, the authors of the paper have being collaborating around the establishment of a federated framework for interoperability of technical applications applied to networked collaborative product development [2]. It was done through participation to or assessment of several research projects in PLM area (RISESTEP - Enterprise Wide standard access to STEP distributed databases -Esprit Project 20459), SAVE (Step in a virtual enterprise- bright euram project 97-5073), OpenDevFactory (Paris cluster Usine Logicielle), CRESCENDO (FP7 Transport 234344 Collaborative and robust engineering using simulation capability enabling next design optimization), TOICA ( Thermal Overall Integrated Conception of Aircraft), SIP@SystemX , Factory of the future area (IMAGINE FoF ICT 201173 Innovative end to end management of DMN), in enterprise application interoperability area (IDEAS IST 2001 37368, ATHENA FP6 IST 507849 Advanced technologies for interoperability of heterogeneous enterprise networks and their applications - COIN Collaboration and interoperability for networked enterprises IST FP7 IST IP project 216256, NEFFICS Networked enterprise transformation and resource management in future internet enabled innovation cloud FP7 ICT 258076) or in Digital Business Ecosystems (FP6 Integrated Project IST-2002-507953).

The assessment through operational projects of approaches coming from Enterprise Application Interoperability domain in one hand, PLM for manufacturing within a System Engineering context in the other hand, demonstrated some drawbacks, with identification of important brakes [2] for industrial usage of manufacturing PLM standards.

PLM interoperability in DMN involved in the development of complex systems requires an effective combination of standards coming from vertical, horizontal and ICT domains, as defined by Object Management Architecture. It also requires effective combination of enablers such as ontology, model driven/service oriented architecture and enterprise modeling, coupled with Model Based System Engineering, Computer Aided Design, Computer Aided Manufacturing and Computer Aided Support. Creating such effective combination of standards is a scientific challenge, due to the silos induced by each concerned community using heterogeneous technologies, languages and paradigms. Applying such effective combination of standards within a DMN is also a challenge because of the uncontrolled evolution of the technologies and solutions developed by each domain or used by each stakeholder.

In order addressing such issues, we have been developing a federative framework for eBusiness PLM interoperability within DMN. We integrated within this framework the Open Group's ArchiMate open and independent standardized enterprise modeling language, which supports the description, analysis and visualization of architecture within and across business domains in an unambiguous way. This choice was made 
after assessing other enterprise modeling standards, and was motivated by the simplicity of the language, its alignment with principles of governance of the evolution of the information system defined by enterprise modeling and control urbanism of enterprise information system, its intent of use for facilitating the communication between enterprise, process, information system and ICT architects, and the existence of the Archi modeling tool. Archi modeling tool (http://www.archimatetool.com) was created by Phil Beauvoir on top of the Eclipse Modeling Tools which implements OMG's specification related to Meta Object Facilities in order supporting Model Driven Architecture. It relies on a formalization of ArchiMate using eCore (http://eclipse.org/modeling/emf), on top of which visual modeling capabilities were develop with full alignment with underlying principles of ArchiMate, i.e. support of multiple views derived from predefined viewpoints associated to precise stakeholders, concerns and authorized subset of language modeling constructs. Archi also provides ability to the user for definition of properties as (name, value) couples, which can be used as a way for annotating an Archi model. In addition, Archi is open source and is an implementation of reference of the open ArchiMate standard, which is a mature standard, as several implementations are available, being on top of commercial or free open source solutions. It makes Archi an appropriate ground for including ArchiMate as a branch of an extended hypermodel for interoperability [3]

\subsection{Problem statement}

Within the last research projects contributing to the establishment of the federated interoperability framework for PLM Interoperability, i.e. IMAGINE and SIP@SystemX, different ways of using Archi have been explored:

- Modeling of the goals, objectives, capabilities, work package, outputs and infrastructure for supporting better communication and decision making between industrial program participants, clients and involved architects and realization teams (e.g. A380 program with about 50 first level sub-contractors and many Airline companies as clients). It is facilitated by the ability to visually represent and interconnect motivation, business, application, technologies, implementation and migration.

- Definition of Blueprint and blueprint templates for design and monitoring of a DMN (IMAGINE), with combination of contract model derived from the decomposition of a product (e.g. Airbus' A380) as a set of configuration items (e.g. SNECMA engine), each configuration item being developed by an enterprise which will be a node of the DMN., partner blueprints, cross organizational collaboration process (e.g. external change management, Technical Data Package secured interchange) blueprints, collaboration capabilities blueprints including collaborative manufacturing PLM hub on the cloud (realized by the cPlatform) and each partner capabilities which are to be involved and interconnected during collaborative product development (e.g. PDM systems of partners for their respective products)

- High level AS IS and TO BE high level representations of interconnected Platform models, Platform Independent Models, Business models and Motivation models 
- Definition of blueprints of standards and underlying framework related to manufacturing PLM (e.g. ISO 10303, ISA 95) and System Engineering standards (e.g. ISO15288), Enterprise process standards (e.g. ArchiMate), Application family (e.g. PDM) standards or ICT standards (e.g. XML technologies), as inputs for being able to define how to jointly combine them all along their lifecycle in order supporting PLM interoperability with a DMN. A fist target is being able to use it for appropriate functional and non-functional specifications by the enterprises for implementation by software solutions, testing, deployment, integration and support. A second target is to support demonstration of appropriate and effective usage for supporting enterprise objectives, goals and processes.

- Definition of a model based approach for assessing PLM standards and their implementation through a test based approach, on top of a test bed which will allow in a first phase to simulate the expected collaboration in a DMN using the standards for targeted business collaboration scenarios, and in a second phase to validate implementations of the standards for supporting these scenarios reusing simulation process, scenarios and data for unitary and integration testing. Implementations of the standards concerns as well software products, applications, methods and business processes within the DMN, and between the partners, their applications and their specific business processes

Doing so, several issues arose concerning usage of model based enterprise modeling relying on Archi within PLM interoperability within System Engineering and manufacturing DMN context.

First ArchiMate scope and intent of use is not the same than other standardized languages such as UML used for Software development, BPMN used for Business Process modeling or XPDL used for design of workflow models and their execution on workflow engines. It's the reason why methods and tools build on top of Archi and enterprise modelling are to be interconnected with methods and tools relying on such standardized languages and associated modeling languages and platforms, implying to properly manage the produced models as consistent sets of artefacts, their relationships with used modeling platform and targeted enterprise execution platforms.

Second Archi is a modeling tool built on top of Eclipse that can be used only on a personal desktop. A single model is produced within a single XML file, but with multiple views. No capabilities exist allowing combining several model trunks, produced by different persons or tools (e.g. self-describing enterprise applications). Or the different blueprint models for support of a DMN have to be produced independently by several organizations and tools as separate artefacts, using ArchiMate with Archi, but also other languages and related modeling tools. As a consequence, as for a manufactured Product, Enterprise data management solution, similar to Product Data Management solutions, are required for collaborative production and consumption of Enterprise models.

Third the enterprise applications underlying conceptual models and the enterprise modeling language are not based on the same meta model and on the same semantic. They are also not implemented on the same technologies: implementation languages for the applications, including programming and serialization languages for applications, including modeling meta-language, notations but also programming and serialization languages of the underlying modeling platforms. As a consequence, the 
model transformation chain is complex when willing to support Model Based Enterprise Collaboration engineering with proper technical data package interchange within a DMN.

This paper describes a new approach for resolving the identify issues.

\section{State of the art and state of the practice}

\subsection{State of the art combined with technologies assessment}

[4] presents the NEFFICS platform which combines an open innovation social media platform with a business modelling and operations platform, and provides a foundation for cloud based open business model innovation, process innovation and service innovation for networked enterprises. It combines usage of Value Delivery Modeling Language (VDML), of the Business Process Model and Notation (BPMN), of the Case Management Model and Notation (CMMN) and of Service Modelling Language (ServiceML) which extends the Service oriented architecture Modeling Language (SoaML).

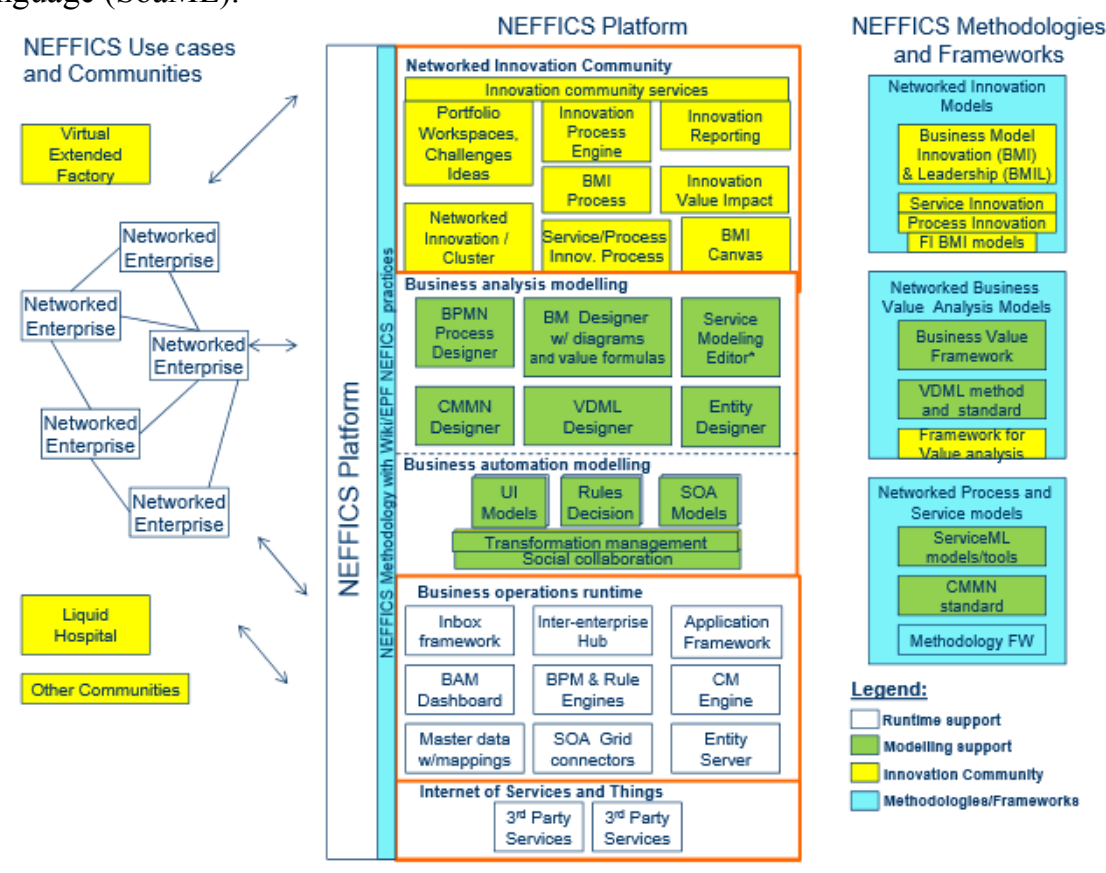

\section{Figure 1: The NEFFICS platform}

Analyzing the NEFFICS architecture as described by Figure 1, the platform appears as innovate platform embedding extended business process designers and dedicated engine. What is targeted as collaboration execution platform is integration of standardized enterprise application components, such as Enterprise Service Bus, respectively Enterprise Workflow System, Enterprise Portal, Enterprise Application 
Server, and Enterprise repository, based on a consistent set of mature open standards, respectively Java Business Integration, Wfmc'XPDL, Enterprise Java beans and LDAP, for which numerous interchangeable COTS are available on the market. The aim is ability of having interchangeable components, without being specific platform, component or product dependent. As a consequence, NEFFICS platform can't be the target. Due to the fact that both modeling and execution platforms are integrated in NEFFICS, needs for model transformation chain is not addressed in NEFFICS. Such a chain is important in particular for integration of manufacturing PLM standards.

[5] points out interest of model driven software engineering which emphasizes on model as primary artifacts in all phases of software development, from requirements analysis over system design to implementation, deployment, verification and validation. It should allow coping with intrinsic complexity of software intensive systems by raising the level of abstraction, and by hiding the complexity of the underlying technology as much as possible. However it requires installing a sophisticated mechanism of model transformation, which enables a wide range of different automated activities such as translation of models (expressed in different modeling languages), generating code from models, model refinement, model synthesis, model extraction and model refactoring. It proposes EMF refactoring usage in a transformation chain based on the AndroMDA tool for model driven software development. We assessed AndroMDA in ATHENA and OpenDevFactory projects. One issue identified comes from the fact that Platform Models are not provided and that transformation is based on velocity, which is not a model to text solution. Underlying technology is really hidden, making it difficult to establish communication between the functional and ICT infrastructure architects. Transformation and deployment logic is hidden and very difficult to correct or debug when something is going wrong. Finally, creating Platform Independent model from PLM standards is an important manual task. Transforming these standards formalization in Domain Specific Language, as UML profiles or as MOF/EMF based DSL, should allow generating the PIM from models of the PLM and System engineering standards, which include as well domain information models (e.g. ISO STEP application protocols, ISA 95), business processes (e.g. ISO15288, SCOR) or business services (e.g. OMG's PLM Services). An issue here is that formal language for specifying the standards are heterogeneous implementation languages which are not necessarily the one used in the execution platform. E.g. a STEP application protocol is formalized in EXPRESS, while languages considered by the targeted execution platform are based on AndroMDA profiles, which consider Manageable entities, value objects, services (local, remote, web), internal processes and user/machine interface interaction processes. Platform targeted for PLM hub are quite more complex than the one targeted by AndroMDA, and being able capturing infrastructure as visual model with appropriate DSL is an important need. Finally, hiding the infrastructure as aimed by model software driven engineering is not accurate when willing ensuring that infrastructure fits with enterprise motivation and when willing to deal appropriately with deployment and evolution.

[6] puts the emphasis on Sirius, a Domain Specific Model graphical editor, which simplifies the production of graphical editing tools for domain specific language, which respond to the previously identified need. So we assess Sirius which is now integrated on OBEO designer, an open source tool based on Eclipse Modeling Tools. 
One drawback we identify comes from the fact the graphical DSL editors are generated on top of Eclipse as execution Platform, and not on top of Web solution. The need exists for publishing a referential of models for supporting effective collaboration, and eventually ability of designing and monitoring the collaboration using graphical modeling and monitoring tools on the web using the created DSL notation.

[7] reports on the TEAP (TOGAF Enterprise Architecture Platform) experience to target Model Driven Organization (MDO) limitations in an industrial context while identifying relevant improvements to the MDE techniques themselves. The focus is on federation of heterogeneous data sources to integrate relevant Enterprise Architecture (EA) information, more easy adaptation of an EA standard to a client needs, with traceability of the different usages, and finally support of multiple views/viewpoints over the same EA repository. The proposed approach indicates that solutions will be made available, which will enrich the Eclipse Modeling Tools for collaborative modeling and federation of models. However TEAP does not address model driven software engineering, and doesn't establish links with PLM standards within a DMN.

\section{Proposition and case study}

In this part, we will introduce the model based approach for establishing PLM interoperability within a DMN, following by a case study.

\subsection{Model of reference for the collaborative PLM hub}

Unlike the other platforms proposed for enterprise interoperability, we propose a simplified reference model in Archi capturing the principle of collaboration supported by the collaborative platform and the applicative components which will be required for the collaborative platform acting as a PLM hub. It includes applicative components for delivering horizontal services usually provided by standardized enterprise solutions (enterprise portal, enterprise service bus, enterprise workflow, enterprise application servers), as well as vertical services related to PLM: PDM repositories and transformation services related to technological frameworks associated to PDM standards: STEP, XML or UML for model exchange, web service and BPEL for distributed Web services and their composition, in order being able to interconnect distributed systems implementing OMG's or OASIS's PLM services. Such generic platform should support PLM business collaborations around a configuration item of an integrated product, for which a component will be provided by a provider, implying exchange of different kind of work orders with associated technical data package. The technical data package will contain product and process data and associated metadata. Figure 2 is a view combining a blueprint template for collaboration, associated to a generic applicative model of the cPlatform. 


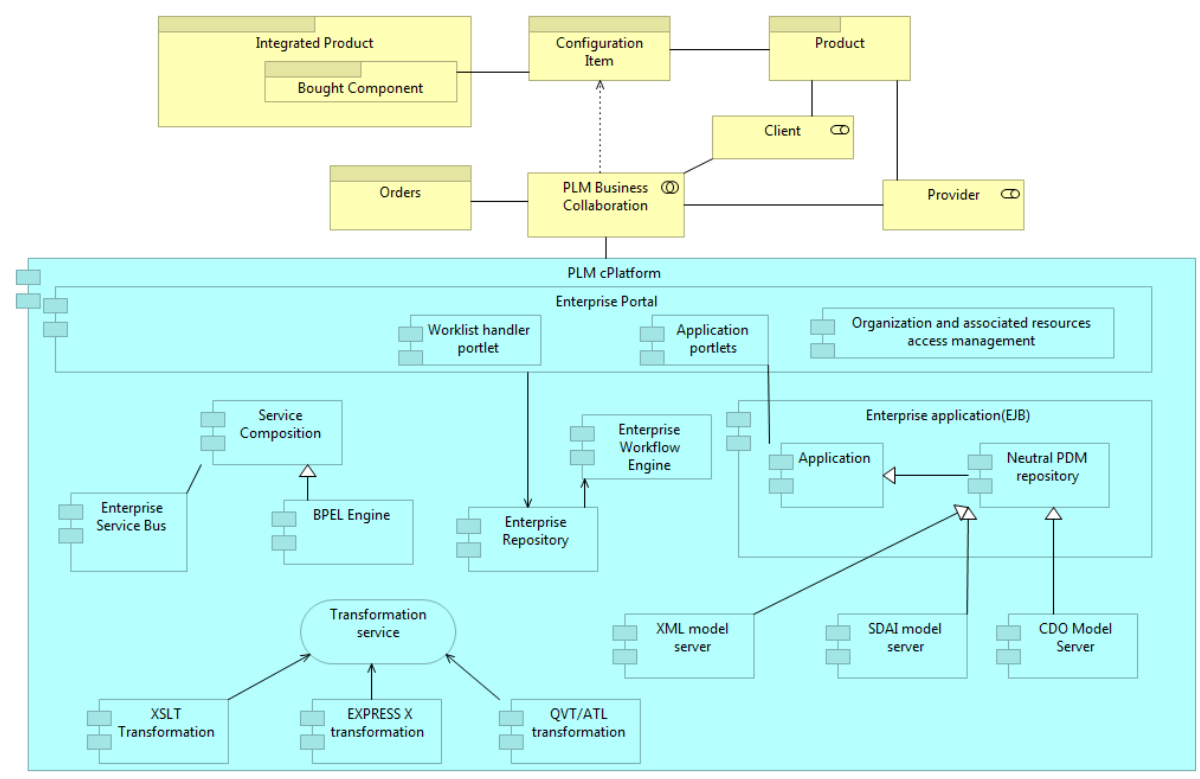

Figure 2: ArchiMate model for cPlatform and supported collaboration

Each applicative component is associated to an open mature standard; all the selected standards have been chosen in order allowing their combination in order covering all the interoperability needs.

It is expected that each application (e.g. PDM system) of the partners involved in the collaboration will rely on standardized service contract elected by the considered digital business ecosystem, here Aeronautic, Space and Defense. Figure 3 is a view capturing the fact that PDM systems of the partners are interconnected through standardized contract, the OMG's PLM services.

The composition of the services is to be realized through cross organizational workflow process models enacted on the enterprise workflow model. Client's and Provider' PDM are (automate) participants of the workflow model, and the tasks they have to realize consist in invocation of operations defined in the PLM standard. Inputs and outputs for the operations are typed according PLM standard for data exchange and sharing, STEP within the aeronautic context.

The cross organization process workflow process is formalized by mean of an orchestration model. If BPMN 2.0 allows modeling orchestration models, it also supports modeling of conversation, collaboration and choreography that can't be executed. However, unlike Wfmc specifications, no architecture of reference is provided with BPMN. Wfmc provides XML process Definition language, which can be distributed between workflow designers and workflow engines provided by different software providers. In addition, since version 2, XPDL was extended with BPMN notation. In addition, only XPDL includes in the meta model task which are to be distributed to work list handlers or to applications. 


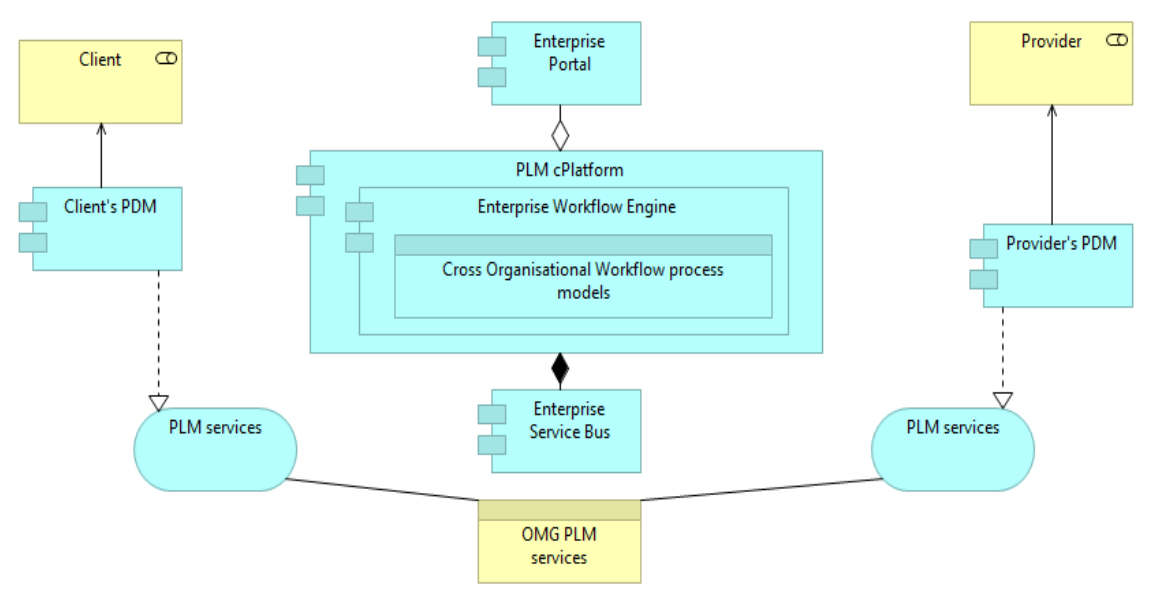

Figure 3: applications plugged on the cPlatform

If ArchiMate modeling constructs include processes, activity and task are not ArchiMate constructs. For such a reason, when willing defining more on details the collaboration processes, the link must be established with XPDL process models a consistent way. It can be done first by associating workflow process model data object and related artefact at ICT layer with the XPDL model defining a generic collaboration process: all the participants are roles defined in the ArchiMate model. Eventually, workflow process activity model can also be captured in ArchiMate, with a detailed view and by typing activities in the view as XPDL: activity. So some complementary constraints are to be put on the view in order ensuring alignment with the XPDL representation (hypermodel for interoperability approach).

The same approach is to be applied with services and data objects. Description of services with ArchiMate doesn't support capture of operations, which is a too low grain of detail. Description of data doesn't support capture of entities. So it will be needed associating services and data objects in Archi with representation based on other languages, supporting the appropriate level of detail. It can be UML for software design (deployment and component diagrams), EXPRESS, XML Schema, Json or EJB entities for data serialization, WSDL for web services, etc. Similarly, ICT layer can be mapped with underlying network and eventually virtualization servers (e.g. ProxMox) allowing automated generation of virtual network or creation of views querying the virtualization servers. At business layer, such mapping can be done with models using more rich languages such as BPMN, IDEF0 or SPEM. For motivation views, it can be mapped with more detailed decision models. Finally implementation and migration views can be related to project planning models. Archi model can be use as integration model dedicated to communication between the different architects and stakeholders, in order aligning CIM, PIM and PM models.

Two model transformation chains, one for software artefacts generation and deployment on the cPlatform, based on solution such as AndroMDA, one for model to model generation and DSL integration, based on solution such as Obeo Designer. 


\subsection{Blueprint template for manufacturing PLM standards}

Numerous types of manufacturing PLM standards have been produced, providing several frameworks for system engineering processes, product data exchange, sharing and long term archiving, PLM services, CAD services, etc.

Such processes don't aim at standardizing the business processes or the software tools. It is the reason why important work is still to be done when willing to implement them and to use them in operational context, as the link is to be made with the specific context, i.e. the actual processes and operational platforms.

In order facilitating assessment and implementation of the standards, our approach allows to produce blueprints describing the standards in order helping each stakeholders, and in particular the architects referenced in the ArchiMate specification (enterprise, process, information system, ICT technologies architects), to better understand how the proposed standards can be use. It also supports defining how the standards can be use together.

Such exercise has been performed on manufacturing standards for product and process data exchange, such as STEP and ISA95. Such standards adopted functional analysis in order capturing information flows between functions. Doing so, no process model is provided (orchestration). Activities of functional model can be mapped with activities of an orchestration model, and then associated to the task that will be distributed in order performing the activity. While relevant workflow data are exchanged between workflow engines and work list handler, application data flow is between workflow participants is not ensured by such system. As the definition of the actual participants is defined dynamically, the need for exchanging or sharing data is known at the very last time. The method for making data available between participants is then to be adapted to the context. In addition, data flow between functions is in fact to be mapped with data flow between participants realizing the tasks. The exception is for control flows, which will correspond to exchange between the participants and the workflow engine that control the distribution of task.

Here again, model transformation will be required for customizing the workflow systems and the data transportation services from data flow provided in such standards, e.g. from Application Activity Model in STEP application protocols, which is formalized using IDEF0. Similarly, ISA 95 provides the description of data flow between function without using a standardized language. This description was very easy to capture with Archi.

Similarly, process framework such as ISO15288 can be easily captured, as illustrated in Figure 4. Such model can be used in order to produce cartography where usage of standards can be contextualized, as illustrated in

Such models are inputs for model transformation which will allow to structure the workflow models of the cPlatform, and which will help the collaboration to rely on process of reference.

Other usage is to provide blueprint models for high level architecture of used technical solutions, of the ICT infrastructure or of the migration from an AS IS solution to a TO BE solution which will deal with ensuring continuous interoperability at an acceptable price. 


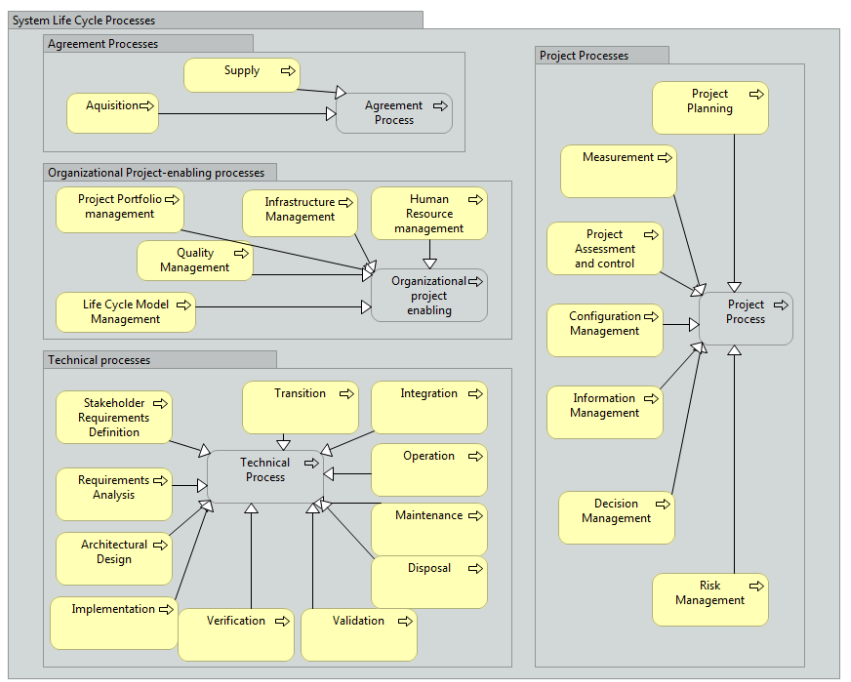

Figure 4: ISO 15288 high level processes

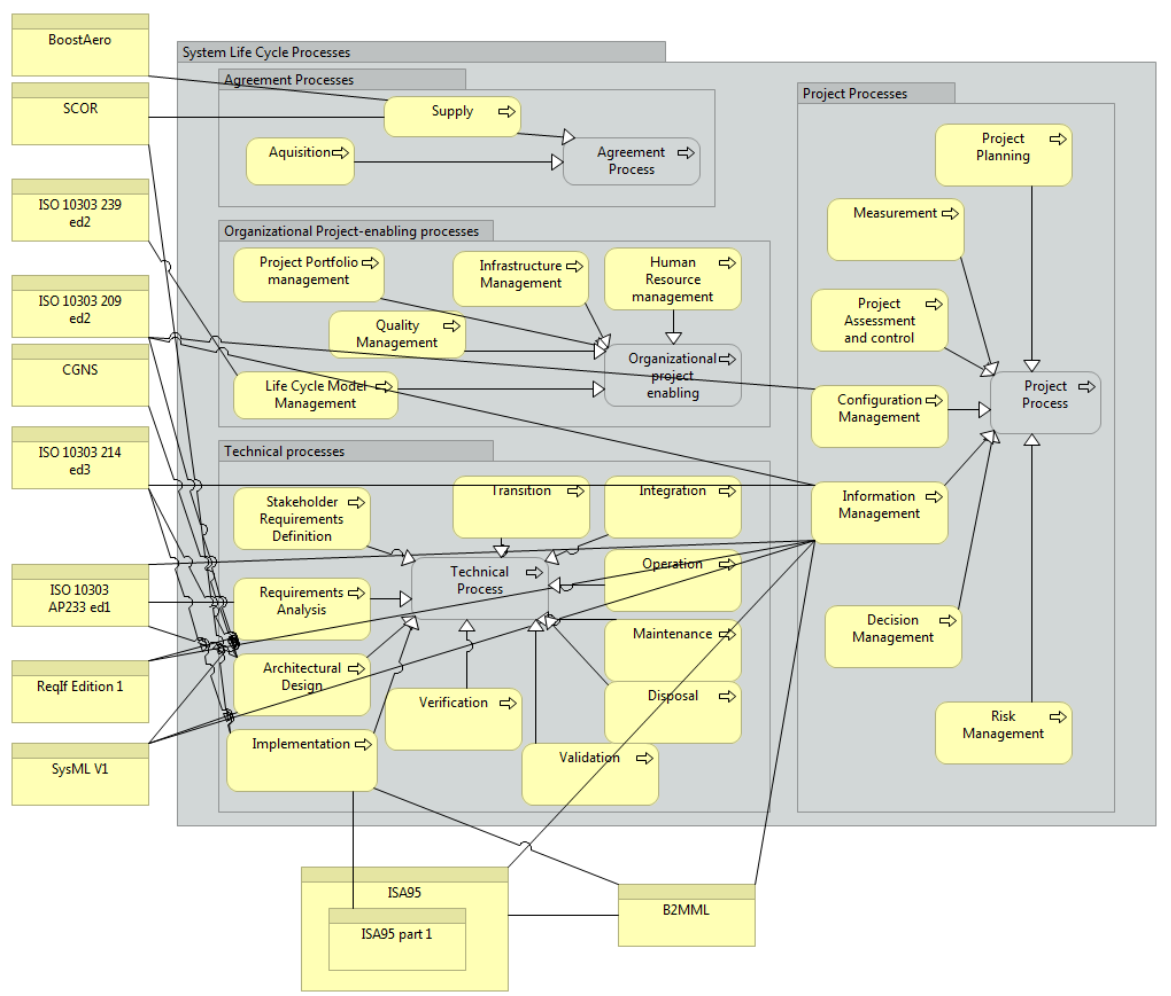

Figure 5: ASD SSG elected standards mapped with ISO 15288 


\section{Conclusion and future work}

In order preparing and building operational PLM interoperability within DMN, this paper proposes an innovative model driven approach which combines enterprise modeling, business modeling, information system modeling and ICT modeling. This approach has been developed through the IMAGINE project for DMN and the SIP@SystemX project, which aims at assessing PLM standards and their implementation on top of a test bed platform based on the cPlatform, and allowing to model use case, test scenarios and to managed them with test data in configuration. The future research in SIP will address simulation of DMN infrastructure and applications for industry being able to properly specify and prepare tests related to implementation of PLM standards to the software vendors, integrators and ICT departments, in alignment with enterprise strategy, processes and methods.

\section{References}

1. Product Lifecycle Management (PLM) Definition, CIMDATA 2009, at https://www.cimdata.com/en/resources/about-plm

2. "Innovative Interoperability Framework for Enterprise Applications within Virtual Enterprises", N Figay, P Ghodous, MEDES 2009

3. "Extended hyper model for interoperability within the Virtual Enterprise", N. Figay, P. Ghodous, accepted for publication at SITIS 2009

4. A.-J. Berre, H. de Man, Y. Lew, B. Elvesæter, and B. M. Ursin-Holm, "Open Business Model, Process and Service Innovation with VDML and ServiceML", in Proc. of the Standardisation for interoperability in the service-oriented enterprise workshop, IWEI 2013

5. Gabriele Taentzer, Dirk Muller, Tom Mens, 2014 Specifying Domain-Specific Refactorings for AndroMDA Based on Graph Transformation. In Applications of Graph Transformations with Industrial Relevance http://dx.doi.org/10.1007/978-3-540-89020-1_9

6. V. Vivyovic, M Maksimovic, B. Perisic, 2014 Sirius: a rapid developmet of DSM graphical editor, in Intelligent Engineering System, 2014 18th International Conference on Intelligent Engineering System

7. Bruneliere Hugo, J. Cabot, S. Frapeau, F. Somda, X. Piers, J. David, C. Calle, J-C Lafaurie, MDE support for enterprise architecture in an industrial context: the TEAP framework Experience, at AMINO 2013, US6. V. Vivyovic, M Maksimovic, B. Perisic, 2014 Sirius: a rapid development of DSM graphical editor, in Intelligent Engineering System, 2014 18th International Conference on Intelligent Engineering System 